\title{
Distinct role for c-kit receptor tyrosine kinase and SgIGSF adhesion molecule in attachment of mast cells to fibroblasts
}

\author{
Yu-ichiro Koma ${ }^{1,2}$, Akihiko Ito ${ }^{1,2}$, Kenji Watabe ${ }^{1}$, Tatsumi Hirata ${ }^{3}$, Masao Mizuki ${ }^{4}$, \\ Hiroshi Yokozaki ${ }^{2}$, Toshio Kitamura ${ }^{5}$, Yuzuru Kanakura ${ }^{4}$ and Yukihiko Kitamura ${ }^{1}$ \\ ${ }^{1}$ Department of Pathology, Osaka University Medical School/Graduate School of Frontier Bioscience, Suita, \\ Osaka, Japan; ${ }^{2}$ Division of Surgical Pathology, Kobe University Graduate School of Medicine, Chuo-ku, Kobe, \\ Japan; ${ }^{3}$ Division of Brain Function, National Institute of Genetics, Graduate University for Advanced Studies, \\ Mishima, Shizuoka, Japan; ${ }^{4}$ Department of Hematology and Oncology, Osaka University Medical School, \\ Suita, Osaka, Japan and ${ }^{5}$ Department of Cell Therapy, Advanced Clinical Research Center, Institute of \\ Medical Science, University of Tokyo, Tokyo, Japan
}

\begin{abstract}
Binding of stem cell factor (SCF) to c-kit receptor tyrosine kinase (KIT) transduces signals essential for mast cell development via several pathways including activation of phosphatidylinositol 3-kinase (PI3-K). When cultured mast cells (CMCs) are cocultured with fibroblasts expressing membrane-bound SCF, CMCs with normal KIT adhere to fibroblasts and proliferate, whereas CMCs lacking cell surface expression of KIT do neither. Spermatogenic immunoglobulin superfamily (SgIGSF) was identified as another molecule that participates in mast cell adhesion to fibroblasts. Since the IC-2 mast cell line expressed neither KIT nor SgIGSF, the effect of ectopic expression of KIT or SgIGSF on the adhesion of IC-2 cells was examined. Three forms of KIT with the normal ectodomain were used: wild-type (KIT-WT) and two mutant types with a phenylalanine substitution at the tyrosine residue 719 (KIT-Y719F) or 821 (KIT-Y821F). KIT-Y719F does not activate PI3-K, whereas KIT-Y821F does. Firstly, KIT or SgIGSF was expressed singly in IC-2 cells. All three forms of KIT increased the adhesion level of IC-2 cells, whereas SgIGSF did not. Secondly, SgIGSF was coexpressed with one of the three forms of KIT. Coexpression of SgIGSF with KIT-WT or KIT-Y821F increased the adhesion level more markedly than was achieved by KIT-WT or KIT-Y821F alone. The effect was abolished by an antibody that blocks SCF-KIT interaction. In contrast, coexpression of SgIGSF with KIT-Y719F did not increase the adhesion level induced by KIT-Y719F alone. In adhesion of mast cells to fibroblasts, KIT appeared to behave as an adhesion molecule and as an activator of other adhesion molecules through phosphorylating PI3-K.
\end{abstract}

Laboratory Investigation (2005) 85, 426-435, advance online publication, 17 January 2005; doi:10.1038/labinvest.3700231

Keywords: immunoglobulin superfamily; KIT tyrosine 719; MITF; phosphatidylinositol 3'-kinase; stromal cells

There are several murine loci that affect the development of mast cells, ${ }^{1,2}$ among the most wellknown are $W$ and Sl. The $W$ locus encodes c-kit receptor tyrosine kinase (KIT),,$^{3,4}$ a growth-factor receptor that is expressed on mast cells, ${ }^{5}$ and the $S 1$ locus encodes stem cell factor (SCF), a cognate ligand of KIT that is produced by several cell types including fibroblasts. ${ }^{6-10}$ SCF exerts its activities either as membrane-bound proteins or as proteoly-

Correspondence: Dr A Ito, MD, Division of Surgical Pathology, Kobe University Graduate School of Medicine, Kusunoki-cho 7-5-1, Chuo-ku, Kobe 650-0017, Japan.

E-mail: akito@med.kobe-u.ac.jp

Received 16 June 2004; revised 29 November 2004; accepted 30 November 2004; published online 17 January 2005 tically processed soluble proteins. ${ }^{11}$ Systemic mast cell deficiency of mice with mutations at the $W$ or $S l$ locus implies that the signals produced by binding of SCF to KIT are essential for development of mast cells.

The important role for SCF-KIT interaction in proliferation of mast cells was reproduced in an in vitro coculture model. ${ }^{12}$ When cultured mast cells (CMCs) were cocultured on a monolayer of NIH/3T3 fibroblasts, CMCs derived from wild-type $(+/+)$ mice proliferated without any specific growth factors. ${ }^{13,14}$ However, when $+/+$ CMCs were prevented from being in contact with NIH/3T3 fibroblasts, they gradually disappeared from the coculture. ${ }^{13}$ In this coculture, adhesion of CMCs to NIH/3T3 fibroblasts appeared to be important for 
CMCs to proliferate, probably because the adhesion allowed KIT on CMCs to bind to membrane-bound SCF expressing on NIH/3T3 fibroblasts.

Adhesion of CMCs to fibroblasts was examined in this coculture. Two mutant alleles were used: the mutant $W$ allele results in lack of cell surface expression of KIT, ${ }^{15,16}$ and the mutant $S 1$ allele is a deletion of the $S$ l locus. ${ }^{6-9}$ CMCs were established from WB- $+/+$ and WB- $W / W$ mice, and fibroblasts from WB-Sl/SI mice. In the coculture, a considerable number of WB-+/+ CMCs adhered to NIH/3T3 fibroblasts, whereas significantly fewer WB- $W / W$ CMCs did. On the other hand, when the cocultures were developed with WB-SI/Sl fibroblasts instead of NIH/3T3 cells, even WB- $/+$ CMCs did not adhere. KIT appeared not merely to transduce growth signals but also to participate in adhesion of CMCs to fibroblasts.

The mi locus also affects mast cell development. ${ }^{17-20}$ Mice with mutations at this locus lack mast cells in various tissues other than the dermis. The mi locus encodes the microphthalmia transcription factor (MITF), a member of the basic-helix-loophelix-leucine zipper family. ${ }^{21,22}$ The mutant $t g$ allele is a transgene-insertional mutation in the $5^{\prime}$ flanking region of the MITF gene. ${ }^{21,23}$ Although the coding region of the MITF gene in C57BL/6 (B6)- $t g / t g$ mice is normal, expression of MITF was not detectable in B6-tg/tg CMCs. ${ }^{24}$ Interestingly, when B6-tg/tg CMCs were cocultured with NIH/3T3 fibroblasts, they showed a poor adhesion. ${ }^{25}$ We found that B6- $+/+$ CMCs abundantly expressed spermatogenic immunoglobulin superfamily (SgIGSF), whereas B6- $\mathrm{tg} / \mathrm{tg}$ CMCs did not express it. Since transfection with SgIGSF cDNA normalized the adhesion of B6- $\mathrm{tg} / \mathrm{tg}$ CMCs, SgIGSF appeared to play an important role in the adhesion of CMCs to fibroblasts.

Although both KIT and SgIGSF belong to the immunoglobulin superfamily, KIT is categorized as a growth-factor receptor and SgIGSF as an adhesion molecule based on the cytoplasmic structural features of each molecule. KIT has five immunoglobulin-like motifs in the extracellular domain and an intracellular domain including a juxtamembrane segment, a kinase divided into two subdomains by a kinase insert sequence, and a carboxy-terminal segment. ${ }^{26-28}$ SgIGSF has three immunoglobulin-like motifs in the extracellular domain and an intracellular domain containing a motif sequence that connects to the actin cytoskeleton. ${ }^{29,30}$ Structurally, KIT resembles colony-stimulating factor-1 (CSF-1) and platelet-derived growth factor receptors, ${ }^{27,28}$ while SgIGSF resembles neural cell adhesion molecule-1 and $-2{ }^{29,30}$ Thus, KIT and SgIGSF may have distinct roles in the adhesion of mast cells to NIH/ 3T3 fibroblasts as a growth-factor receptor and as an adhesion molecule, respectively.

In the present study, we first screened expression of KIT and SgIGSF in various types of CMCs and mast cell lines, and then evaluated the adhesion of these cells to NIH/3T3 fibroblasts. We found that IC-2 cells, an interleukin-3-dependent mast cell line, ${ }^{31}$ expressed neither KIT nor SgIGSF and showed the lowest level of adhesion. We then established IC-2 cells that ectopically expressed either KIT or SgIGSF, or both, and evaluated their adhesion. KIT-mediated intracellular signals appeared to be necessary for SgIGSF to mediate the adhesion of IC-2 cells to NIH/ 3T3 fibroblasts.

\section{Materials and methods}

\section{Mice}

The WB- $+/+$ and WB- $W / W$ mice were purchased from Japan SLC (Hamamatsu, Japan). VGA-9-tg/tg mice were kindly provided by $\mathrm{Dr} \mathrm{H}$ Arnheiter (National Institutes of Health, Bethesda, MD, USA). The VGA-9-tg/tg mice were maintained by consecutive back-crosses to our own inbred WB colony more than 12 generations. WB-tg/tg mice were produced by crosses between the female and male heterozygotes and selected by their white coat color.

\section{Cells}

Pokeweed mitogen-stimulated spleen cell-conditioned medium (PWM-SCM) was prepared as described previously. ${ }^{25}$ Spleen cells derived from 2- to 3 -week-old mice were cultured in $\alpha$-MEM (ICN Biomedicals, Costa Mesa, CA, USA) supplemented with 10\% PWM-SCM and 10\% FCS (Nippon Biosupply Center, Tokyo, Japan). Half of the medium was replaced every 7 days. After 4 weeks, more than $95 \%$ of the cells were CMCs. CMCs and two mast cell lines, IC-2 and MC/9, ${ }^{31,32}$ were grown in $\alpha$ MEM supplemented with 10\% PWM-SCM and 10\% FCS. Two mastocytoma cell lines, FMA/3 and P$815,{ }^{33,34}$ were grown in $\alpha$-MEM supplemented with $10 \%$ FCS. Another mastocytoma cell line, MST, ${ }^{35}$ was kindly provided by Dr JD Esko (University of California, San Diego, CA, USA), and grown in RPMI 1640 (Sigma Chemical Co., St Louis, MO, USA) supplemented with $10 \%$ FCS. NIH/3T3 fibroblasts and $\Psi 2$ retrovirus packaging cells were maintained in DMEM (ICN Biomedicals) supplemented with $10 \%$ FCS $^{36}$ Plat-E retrovirus packaging cells $^{37}$ were maintained in DMEM supplemented with $10 \%$ FCS, $1 \mu \mathrm{g} / \mathrm{ml}$ puromycin (Sigma), and $10 \mu \mathrm{g} / \mathrm{ml}$ blasticidin S (Invitrogen, Carlsbad, CA, USA).

\section{Antibodies}

The anti-SgIGSF rabbit polyclonal antibody (Ab) was generated according to the method described by Wakayama et al. $^{38}$ The anti-KIT armenian hamster monoclonal $\mathrm{Ab}(\mathrm{H} 2 \mathrm{C} 7)$ was generated previously and used for immunocytochemistry. ${ }^{39}$ Other primary Abs were anti-KIT rabbit polyclonal (used for 
Western blot analyses; Dako, Kyoto, Japan), anti-KIT rat monoclonal (used for adhesion assays; ACK45; Pharmingen, San Diego, CA, USA), anti-actin mouse monoclonal (AC-40; Sigma), anti- $\alpha 5$ integrin rabbit polyclonal (Chemicon International, Temecula, CA, USA) and anti- $\beta 1$ integrin mouse monoclonal Abs (Transduction Laboratories, Lexington, KY, USA). Secondary Abs used were peroxidase-conjugated anti-rabbit or anti-mouse IgG Abs (MBL, Nagoya, Japan), and Cy2-conjugated anti-rabbit or Cy3-conjugated anti-armenian hamster IgG Abs (Jackson Immunoresearch Laboratories, West Grove, PA, USA).

\section{Western Blot Analysis}

Cells were lysed in a buffer containing $50 \mathrm{mM}$ Tris$\mathrm{HCl}$ (pH 8.0), $150 \mathrm{mM} \mathrm{NaCl}, 1 \%$ Triton X-100, and $1 \%$ protease inhibitor cocktail (Sigma). The resulting lysates were separated on $10 \%$ SDS-polyacrylamide gels, transferred to Immobilon (Millipore, Bedford, MA, USA), and reacted with the primary Abs indicated. After washing, the blots were incubated with an appropriate peroxidase-labeled secondary $\mathrm{Ab}$ and then reacted with Western Lighting reagents (PerkinElmer Life Sciences, Boston, MA, USA) before exposure. After stripping, the blots were probed with anti-actin Ab.

\section{Adhesion Assay}

Mast or mastocytoma cells were labeled with PKH67-GL (Sigma), a green-fluorescent cell linker dye, according to the manufacturer's instruction. Briefly, cells $\left(5 \times 10^{6} \mathrm{cells} / \mathrm{ml}\right)$ were incubated with the dye $(10 \mu \mathrm{M})$ for $5 \mathrm{~min}$ at room temperature. The dye staining reaction was stopped by addition of two volumes of FCS. The cells were diluted further in two volumes of medium, washed three times, and rested for at least $15 \mathrm{~h}$ at $37^{\circ} \mathrm{C}$. Labeled cells $\left(1.0 \times 10^{5}\right)$ were suspended in $2 \mathrm{ml} \alpha$-MEM containing $10 \%$ FCS and added to a confluent culture of NIH/3T3 fibroblasts in 35-mm culture dishes. After $3 \mathrm{~h}$ of coculture, the dishes were washed five times with warmed $\left(37^{\circ} \mathrm{C}\right)$ PBS (pH 7.4$)$ to remove nonadherent cells. NIH/3T3 fibroblasts and adherent mast or mastocytoma cells were harvested by trypsin treatment and centrifugation, fixed by adding $1 \mathrm{ml}$ of ice-cold $2 \%$ paraformaldehyde in PBS, and analyzed on a FACScan (Becton Dickinson, Franklin Lakes, NJ, USA). At least $1.0 \times 10^{4}$ events were analyzed for each sample. The green-fluorescence intensity-based histogram detected two cell populations, fluorescence-negative (NIH/3T3 fibroblasts) and -positive (labeled mast or mastocytoma cells): cells with intermediate fluorescence intensity were a negligibly small population. Event numbers of the fluorescence-negative and -positive populations were counted by gating either population. The adhesion of labeled cells to NIH/3T3 fibroblasts was quantified by dividing the event number of the fluorescence-positive population by the event number of the fluorescence-negative population, and the obtained value was expressed as the number of adhering cells per NIH/3T3 fibroblast. Triplicate dishes were prepared for each group of coculture, and the mean values and standard errors (s.e.) were calculated. A recombinant protein of the SgIGSF extracellular domain fused with the human $\operatorname{IgG}_{1} \mathrm{FC}_{\mathrm{C}}$ fragment (SgIGSF-ED-FC) was purified as described previously. ${ }^{40}$ In some adhesion experiments, labeled cells were preincubated for $30 \mathrm{~min}$ at $37^{\circ} \mathrm{C}$ with indicated concentrations of SgIGSF-ED-FC or ACK45, and the adhesion assay was performed in the presence of either protein. All experiments were repeated three times with similar results.

\section{Plasmid Construction}

We previously described the plasmid constructs that express wild-type KIT (KIT-WT), or a mutant form of KIT with a phenylalanine substitution of the tyrosine residue 719 (KIT-Y719F) or 821 (KITY821F). ${ }^{41}$ The cDNA inserts were amplified by polymerase chain reaction using Pyrobest Taq DNA polymerase (Takara, Kyoto, Japan) with a primer set: sense, 5'-GAGTCTAGCGCAGCCACCGC GATGAGAGGCGCTCGCG-3' (containing the first codon of KIT); and antisense, $5^{\prime}$-GGTTTCTGCTCAG GCATCTTCGTGCACGAG-3' (containing the stop codon of KIT). The amplified fragments were phosphorylated by T4 Polynucleotide Kinase (Gibco BRL, Grand Island, NY, USA) and inserted directionally into the blunted EcoRI site of the pM5Gneo retroviral vector. ${ }^{42}$

\section{Transfection of Mast Cells with Retrovirus Vector}

The $\Psi 2$ cells that produced high titers of viruses containing SgIGSF cDNA were prepared in our laboratory. ${ }^{25}$ IC-2 cells were cultured on a $\gamma$ irradiated $(30 \mathrm{~Gy})$ subconfluent monolayer of the virus-producing $\Psi 2$ cells for $48 \mathrm{~h}$ in the presence of polybrene $(8 \mu \mathrm{g} / \mathrm{ml}$; Sigma). Then the single IC-2 cells were cultured in the presence of blasticidin $\mathrm{S}$ $(10 \mu \mathrm{g} / \mathrm{ml}$; Invitrogen) for at least 2 weeks to obtain the infected clones. For ectopic expression of various forms of KIT, Plat-E cells were transiently transfected with the pM5Gneo vector expressing KIT-WT, KIT-Y719F, or KIT-Y821F by using Fugene6 reagents (Roche Diagnostics, Indianapolis, IN, USA). After $24 \mathrm{~h}$, cell-free culture supernatants were harvested. IC-2 cells were then incubated with the virus-containing supernatants for $48 \mathrm{~h}$ in the presence of polybrene ( $8 \mu \mathrm{g} / \mathrm{ml}$; Sigma). Infected clones were selected with the resistance to G418 $(0.8 \mathrm{mg} / \mathrm{ml}$; Gibco BRL). The procedure for ectopic expression of SgIGSF in CMCs was described previously. ${ }^{25}$ 


\section{Immunocytochemistry}

To stain the coculture, an NIH/3T3 monolayer was established on a cover slip placed at the bottom of a culture dish and IC-2 cells were plated over this. After 3-h coculture, the cover slips were washed with PBS and fixed with methanol at $-20^{\circ} \mathrm{C}$ for 20 min. Fixed samples were blocked with $2 \%$ BSA in PBS, incubated with the anti-SgIGSF and anti-KIT (H2C7) Abs, and stained with Cy2-conjugated antirabbit IgGAb and Cy3-conjugated anti-armenian hamster IgG Ab, respectively. Cells were visualized using a confocal laser scanning microscope (LSM510; Carl Zeiss, OberKochen, Germany).

\section{Results}

\section{Comparison between the Expression of SgIGSF and} KIT and the Adhesion to NIH/3T3 Fibroblasts

CMCs were obtained by culturing spleen cells of WB- $+/+, \mathrm{WB}-W / W$, and WB-tg/tg mice. Expression of SgIGSF and KIT by these CMCs was examined by Western blotting. Expression levels of SgIGSF were comparable between WB- $+/+$ and $W B-W / W$ CMCs, whereas SgIGSF was not expressed by WB-tg/tg CMCs (Figure 1a). KIT expression was easily detected in the lysate of $\mathrm{WB}-+/+\mathrm{CMCs}$, but not in that of WB-W/W CMCs (Figure 1a). WB-tg/tg CMCs expressed a significantly reduced but appreciable level of KIT (Figure 1a). For reference, we examined expression level of VLA-5, a complex of $\alpha 5$ and $\beta 1$ integrins, and found that it was not reduced in WB-tg/tg CMCs (Figure 1a). Then, adhesion of the three types of CMCs to NIH/3T3 fibroblasts was examined. The adhesion of $\mathrm{WB}-W / W$ and that of WB-tg/tg CMCs were significantly impaired when compared to that of WB- + / CMCs (Table 1), showing involvement of both SgIGSF and KIT in the adhesion of CMCs to NIH/3T3 fibroblasts.

Next, expression levels of SgIGSF and KIT were examined in various mast cell lines. IC-2 and MC/9 cells expressed neither SgIGSF nor KIT (Figure 1b). MST tumor mast cells abundantly expressed KIT but did not express SgIGSF (Figure 1b). FMA3 and P-815 tumor mast cells expressed both SgIGSF and KIT (Figure 1b). KIT expression was detected as a doublet: the band at $145 \mathrm{kDa}$ corresponded to the mature form of KIT and the band at $125 \mathrm{kDa}$ corresponded to the immature form. ${ }^{43}$ The mobility size of SgIGSF was different between FMA3 and P-815 tumor mast cells. This was probably due to cell-type-specific glycosylation of SgIGSF. ${ }^{25}$ The adhesion to NIH/3T3 fibroblasts was compared among the above-mentioned mast cell lines. FMA3 and P-815 tumor mast cells with SgIGSF and KIT expression showed greater adhesion levels than those of IC-2 and MC/9 cells without SgIGSF and KIT expression (Table 1). The adhesion level of MST a

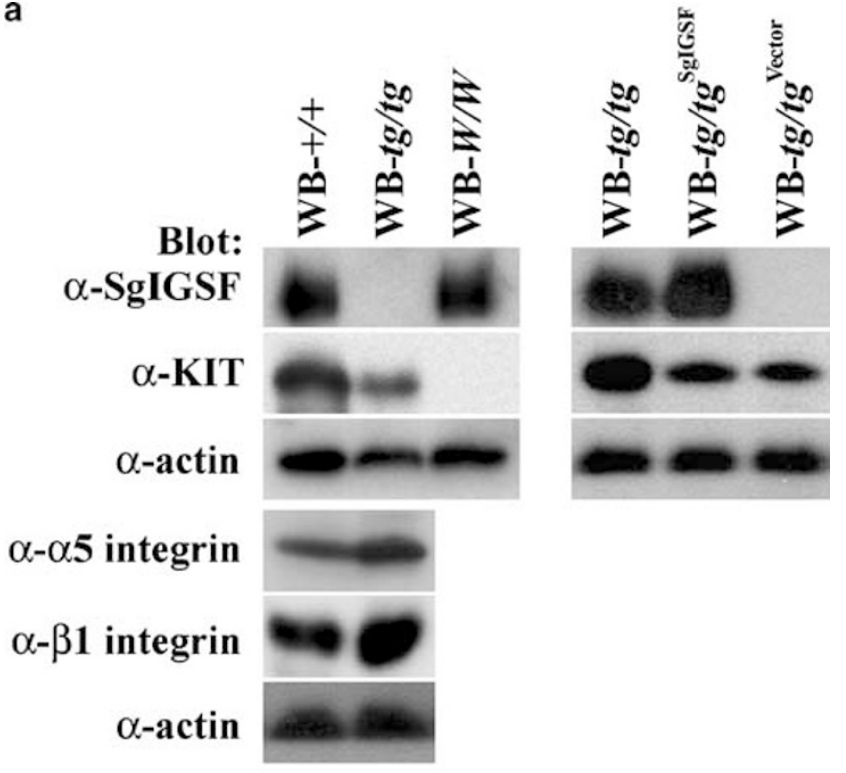

b

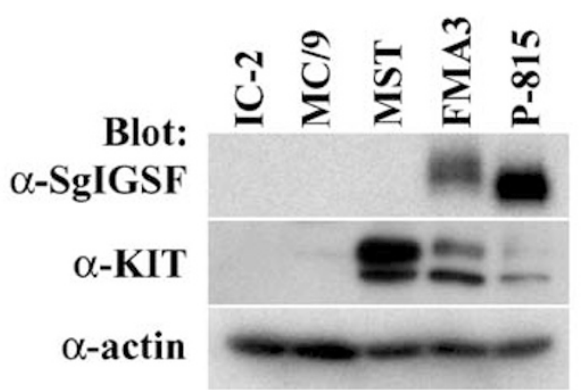

Figure 1 Expression of SgIGSF and KIT in various CMCs and mast cell lines. Protein lysates from CMCs of various genotypes or their transfectants (a) and from various mast or mastocytoma cell lines (b) were electrophoresed and blotted with Abs indicated. After stripping, the blots were probed with the anti-actin Ab to indicate the total amount of proteins loaded per lane.

Table 1 Adhesion of various CMCs and mast cell lines to NIH/ 3 T3 fibroblasts

\begin{tabular}{lc}
\hline Cells & $\begin{array}{c}\text { No. of adhering cells per } \\
\text { NIH/3T3 fibroblast }\end{array}$ \\
\hline WB-+/+CMCs & $0.117 \pm 0.004$ \\
WB-W/W CMCs & $0.053 \pm 0.001^{*}$ \\
WB-tg/tg CMCs & $0.040 \pm 0.001^{*}$ \\
IC-2 & \\
MC/9 & $0.012 \pm 0.001 * *$ \\
MST & $0.037 \pm 0.001 * *$ \\
FMA3 & $0.061 \pm 0.002 * *$ \\
P-815 & $0.073 \pm 0.003 * *$ \\
\end{tabular}

\footnotetext{
${ }^{\mathrm{a}}$ Mean and s.e. of six dishes.

${ }^{*} P<0.01$ by Student's $t$-test when compared with the value of WB-+/+ CMCs.

${ }^{*} P<0.01$ by Student's $t$-test when compared with the values of the other types of cells located in the adjacent line.
}

tumor mast cells expressing KIT alone was intermediate between the adhesion levels of MC/9 and FMA3 cell lines (Table 1). 
Necessity of KIT for SgIGSF to Mediate the Adhesion of IC-2 Cells

IC-2 cells expressed neither SgIGSF nor KIT and showed the lowest level of adhesion to NIH/3T3 fibroblasts. We examined whether ectopic expression of SgIGSF and/or KIT may improve the adhesion level of IC-2 cells. We established three IC-2 clones that expressed SgIGSF alone (IC-2 ${ }^{\text {SgIGSF}}$ ),

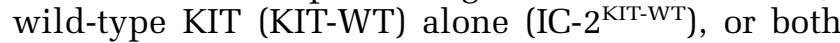

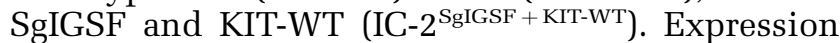
levels of SgIGSF were comparable between IC-2 ${ }^{\text {SgIGSF }}$ and IC-2 ${ }^{\text {SgIGSF + KIT-WT }}$ cells, and expression levels of KIT were comparable between IC-2 ${ }^{\text {KIT-WT }}$ and IC$2^{\text {SgIGSF + KIT-WT }}$ cells (Figure 2a). The adhesion of these three clones was examined by coculturing with NIH/3T3 fibroblasts. Unexpectedly, there was no improvement in the adhesion level of IC- $2^{\text {SgIGSF }}$ cells (Table 2). In contrast, IC-2 ${ }^{\text {KIT-WT }}$ cells showed a significantly greater adhesion level than that of original IC-2 cells (Table 2). The adhesion level of IC-2 ${ }^{\text {SgIGSF + KIT-WT }}$ cells was significantly higher than that of IC-2 ${ }^{\text {KIT-WT }}$ cells (Table 2).

We also examined whether ectopic SgIGSF improved the adhesion level of WB-tg/tg CMCs. WB-tg/ tg CMCs were transfected with SgIGSF cDNA. The obtained CMCs (WB-tg/tg $\mathrm{CMCs}^{\text {SgIGSF}}$ ) expressed SgIGSF a little more abundantly than WB- $+/+$ CMCs (Figure 1a) and adhered normally to NIH/3T3 fibroblasts (Table 2). Vector transfection (WB-tg/tg $\mathrm{CMCs}^{\text {Vector }}$ neither influenced SgIGSF expression nor the adhesion (Figure 1a and Table 2).

Inhibition of the Adhesion of IC-2 ${ }^{\text {SgIGSF + KIT-WT Cells to }}$ NIH/3T3 Fibroblasts by Soluble SgIGSF Ectodomain or Anti-KIT Ab

We examined whether the adhesion of IC-2 ${ }^{\text {SgIGSF + KIT-WT }}$ cells to NIH/3T3 fibroblasts was inhibited by the proteins that bound to SgIGSF or KIT. The soluble extracellular domain of SgIGSF was purified as a fusion protein with the human $\operatorname{IgG}_{1} F_{c}$ fragment (SgIGSF-ED-FC). ${ }^{40}$ Since homophilic binding of SgIGSF had been reported, SgIGSF-ED-Fc appeared to bind membrane-bound SgIGSF expressed on IC$2^{\text {SgIGSF + KIT-WT }}$ cells. SgIGSF-ED-FC was added to the coculture of IC-2 $2^{\text {SgIGSF + KIT-WT }}$ cells with NIH/3T3 fibroblasts at several concentrations ranging from 0.5 to $50 \mu \mathrm{g} / \mathrm{ml}$. SgIGSF-ED-Fc showed a significant inhibitory effect on the adhesion of IC-2 $2^{\text {SgIGSF }+ \text { KIT-WT }}$ cells, although a concentration as high as $50 \mu \mathrm{g} / \mathrm{ml}$ was required (Table 3 ). At this concentration, the adhesion level of IC-2 $2^{\text {SgIGSF + KIT-WT }}$ cells was comparable with that of IC-2 ${ }^{\text {KIT-WT }}$ cells (Table 3 ).

The adhesion level of WB- $+/+$ CMCs was also lowered by SgIGSF-ED-Fc, but was still higher than that of WB- $W / W$ CMCs (Table 3). In contrast, SgIGSF-ED-Fc did not decrease the adhesion level of WB- $W / W$ CMCs at all (Table 3). Control human $\mathrm{IgG}_{1}$ had no effect on the adhesion of IC-2 cells or CMCs (Table 3). a

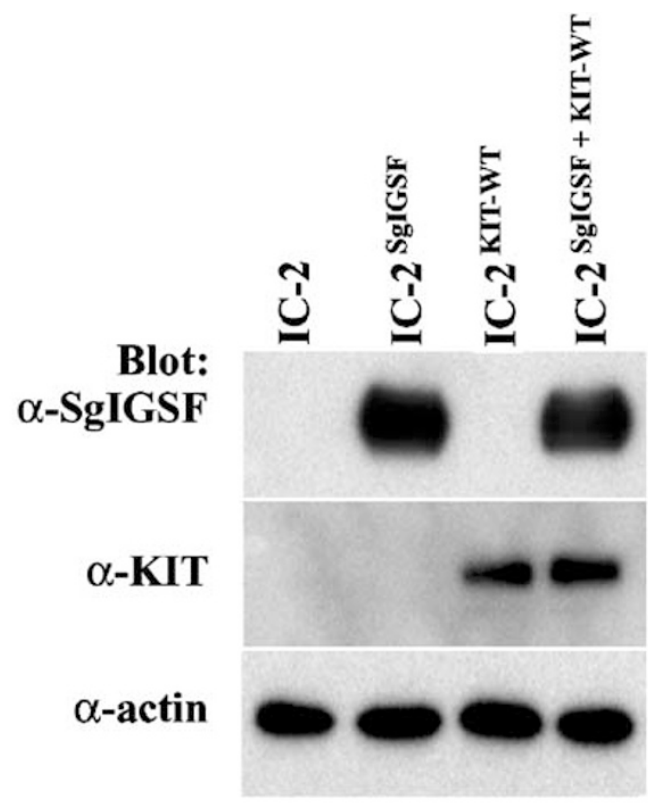

b

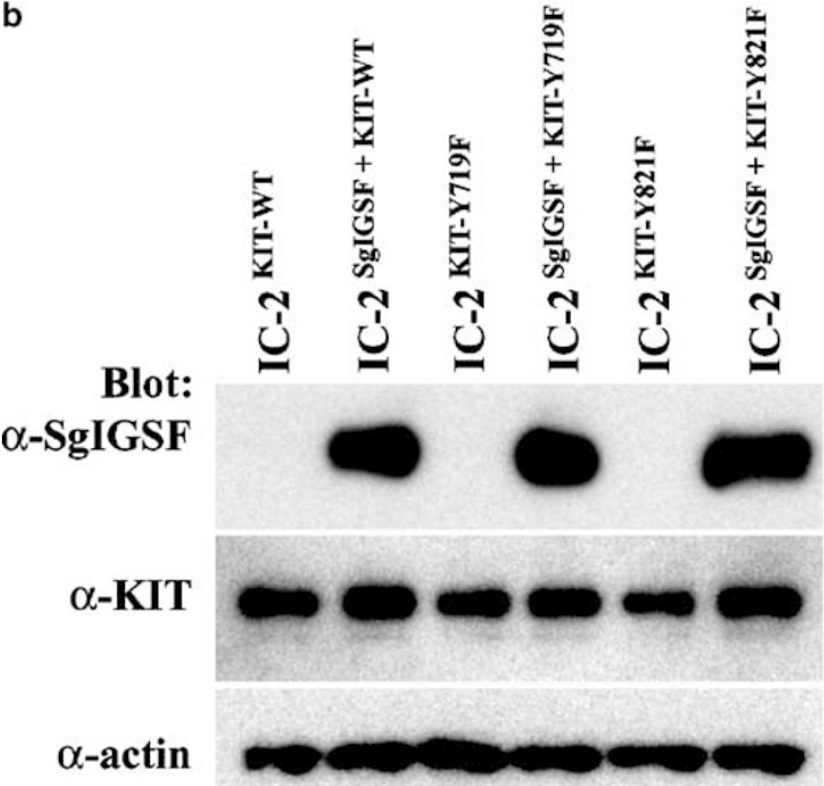

Figure 2 Establishment of various IC-2 cell clones. (a) Western blot analyses of IC-2 cell clones that ectopically express SgIGSF alone, KIT-WT alone, or both SgIGSF and KIT-WT. (b) Western blot analyses of IC-2 cell clones that ectopically express KITY719F or KIT-Y821F together with or without SgIGSF. Protein lysates from various IC-2 cell clones were electrophoresed and blotted with either the anti-SgIGSF or the anti-KIT (rabbit polyclonal) Ab. After stripping, the blots were probed with the anti-actin $\mathrm{Ab}$ to indicate the total amount of proteins loaded per lane.

Next, we examined the adhesion of IC- $2^{\text {SgIGSF }+ \text { KIT-WT }}$ cells in the presence of an anti-KIT Ab, ACK45, that binds to the extracellular domain of KIT and blocks the interaction between KIT and SCF. ${ }^{44,45}$ ACK45 inhibited the adhesion of IC-2 ${ }^{\text {SgIGSF }+ \text { KIT-WT }}$ cells in a dose-dependent manner (Table 4). When the Ab was 
Table 2 Adhesion to NIH/3T3 fibroblasts of IC-2 cells and WB-tg/tg CMCs that express either SgIGSF or KIT, or both

\begin{tabular}{|c|c|}
\hline Cells & $\begin{array}{l}\text { No. of adhering cells per } \\
\text { NIH/3T3 fibroblast }\end{array}$ \\
\hline IC-2 & $0.011 \pm 0.001$ \\
\hline IC- $2^{\text {SgIGSF }}$ & $0.009 \pm 0.001$ \\
\hline IC-2 ${ }^{\text {KIT-WT }}$ & $0.043 \pm 0.002 *$ \\
\hline IC-2 $2^{\text {SgIGSF+KIT-WT }}$ & $0.061 \pm 0.001^{* * * *}$ \\
\hline WB-+/+ CMCs & $0.112 \pm 0.004$ \\
\hline WB-tg/tg CMCs ${ }^{\text {SgIGSF }}$ & $0.120 \pm 0.005$ \\
\hline WB-tg/tg CMCs sector $^{\text {Vet }}$ & $0.043 \pm 0.002 * * *$ \\
\hline
\end{tabular}

${ }^{\mathrm{a}}$ Mean and s.e. of six dishes.

${ }^{*} P<0.01$ by Student's $t$-test when compared with the value of IC- 2 cells.

${ }^{*} P<0.01$ by Student's $t$-test when compared with the value of IC-2 $2^{\text {KIT-WT }}$ cells.

$* * * P<0.01$ by Student's $t$-test when compared with the value of WB-+/+ CMCs.

Table 3 Inhibition of the adhesion of IC-2 $2^{\text {SgIGSF+KIT-WT }}$ cells and WB-+/+ CMCs by SgIGSF-ED-Fc

\begin{tabular}{|c|c|c|c|}
\hline \multirow[t]{2}{*}{ Cells } & \multicolumn{2}{|c|}{$\begin{array}{l}\text { Amount of added protein } \\
\qquad(\mu \mathrm{g} / \mathrm{ml})\end{array}$} & \multirow{2}{*}{$\begin{array}{l}\text { No. of adhering } \\
\text { cells per NIH/3T3 } \\
\text { fibroblast }\end{array}$} \\
\hline & $S g I G S F-E D-F C$ & $h I g G_{1}$ & \\
\hline IC-2 $2^{\text {SgIGSF+KIT-WT }}$ & 0 & 0 & $0.065 \pm 0.001$ \\
\hline IC- $2^{\text {SgIGSF+KIT-WT }}$ & 0.5 & 0 & $0.063 \pm 0.003$ \\
\hline IC- $2^{\text {SgIGSF+KIT-WT }}$ & 5 & 0 & $0.061 \pm 0.003$ \\
\hline IC- $2^{\text {SgIGSF+KIT-WT }}$ & 50 & 0 & $0.054 \pm 0.002^{*}$ \\
\hline IC- $2^{\text {SgIGSF+KIT-WT }}$ & 0 & 50 & $0.066 \pm 0.002$ \\
\hline IC-2 $2^{\text {KIT-WT }}$ & 0 & 0 & $0.048 \pm 0.003^{*}$ \\
\hline WB-+/+ CMCs & 0 & 0 & $0.108 \pm 0.005$ \\
\hline WB-+/+ CMCs & 50 & 0 & $0.075 \pm 0.004^{* *}$ \\
\hline WB-+/+ CMCs & 0 & 50 & $0.102 \pm 0.003$ \\
\hline WB- $W / W$ CMCs & 0 & 0 & $0.056 \pm 0.003$ \\
\hline WB- $W / W$ CMCs & 50 & 0 & $0.053 \pm 0.002$ \\
\hline WB- $W / W$ CMCs & 0 & 50 & $0.055 \pm 0.002$ \\
\hline
\end{tabular}

${ }^{\mathrm{a}}$ Mean and s.e. of six dishes.

${ }^{*} P<0.01$ by Student's $t$-test when compared with the value of IC-2 ${ }^{\text {SgIGSF+KIT-WT }}$ cells in the absence of SgIGSF-ED-FC.

${ }^{*} P<0.01$ by Student's $t$-test when compared with the values of WB-+/+ and WB-W/W CMCs in the absence of SgIGSF-ED-Fc.

added at a concentration of $0.5 \mu \mathrm{g} / \mathrm{ml}$, the adhesion level of IC-2 ${ }^{\text {SgIGSF + KIT-WT }}$ cells was reduced to that of original IC-2 cells (Table 4). Similar inhibitory effect of ACK 45 was detected on the adhesion of IC-2 $2^{\text {KIT-WT }}$ cells (Table 4).

\section{Adhesion of IC-2 Cells that Express Mutant Forms of KIT}

Binding of SCF induces the activation of KIT, and the activated KIT further phosphorylates phosphatidylinositol 3-kinase (PI3-K). ${ }^{46,47}$ Tyrosine residue
Table 4 Inhibition of the adhesion of IC-2 $2^{\text {SgIGSF+KIT-WT }}$ cells by ACK45

\begin{tabular}{llc}
\hline Cells & $\begin{array}{c}\text { ACK45 } \\
(\mu \mathrm{g} / \mathrm{ml})\end{array}$ & $\begin{array}{c}\text { No. of adhering cells per } \\
\text { NIH/3T3 fibroblast }\end{array}$ \\
\hline IC-2 $^{\mathrm{SgIGSF+KIT-WT}}$ & 0 & $0.056 \pm 0.001$ \\
IC-2 $^{\text {SgIGSF+KIT-WT }}$ & 0.005 & $0.048 \pm 0.001$ \\
IC-2 $^{\text {SgIGSF+KIT-WT }}$ & 0.05 & $0.017 \pm 0.001^{*}$ \\
IC-2 $^{\text {SgIGSF+KIT-WT }}$ & 0.5 & $0.011 \pm 0.001^{*}$ \\
IC-2 $^{\text {KIT-WT }}$ & 0 & $0.039 \pm 0.003$ \\
IC-2 $^{\text {KIT-WT }}$ & 0.5 & $0.010 \pm 0.001^{* *}$ \\
IC-2 & 0 & $0.010 \pm 0.001^{*}$ \\
\hline
\end{tabular}

${ }^{\mathrm{a}}$ Mean and s.e. of six dishes.

${ }^{*} P<0.01$ by Student's $t$-test when compared with the value of IC-2 $2^{\text {SgIGSF+KIT-WT }}$ cells in the absence of ACK45.

${ }^{*} P<0.01$ by Student's $t$-test when compared with the value of IC-2 ${ }^{\text {KTT-WT }}$ cells in the absense of ACK45.

Table 5 Adhesion to NIH/3T3 fibroblasts of IC-2 cells that express mutant KIT singly or coexpress it with SgIGSF

\begin{tabular}{lc}
\hline Cells & $\begin{array}{c}\text { No. of adhering cells per } \\
\text { NIH/3T3 fibroblast }\end{array}$ \\
\hline IC-2 & \\
KIT-WT & $0.057 \pm 0.002$ \\
IC-2 $^{\text {KIT-Y719F }}$ & $0.059 \pm 0.001$ \\
IC-2 $^{\text {SgIGSF+KIT-WT }}$ & $0.056 \pm 0.001$ \\
IC-2 $^{\text {SgIGSF+KIT-Y719F }}$ & $0.079 \pm 0.003^{*, * *}$ \\
IC-2 $^{\text {SgIGSF+KIT-Y821F }}$ & $0.057 \pm 0.002$ \\
\end{tabular}

${ }^{\mathrm{a}}$ Mean and s.e. of six dishes.

${ }^{*} P<0.01$ by Student's $t$-test when compared with the value of IC-2 $2^{\text {KIT-WT }}$ cells.

${ }^{*} P<0.01$ by Student's $t$-test when compared with the value of IC-2 ${ }^{\text {KTT-Y821F }}$ cells.

719 is the only PI3-K-binding site of KIT, ${ }^{48}$ whereas tyrosine residue 821 is the homolog of tyrosine residue 809 in CSF-1 receptor, which is essential for CSF-1-induced mitogenic signaling and the induction of $\mathrm{C}-\mathrm{myc}$ expression. ${ }^{49,50}$ Addition of SCF induced the adhesion of CMCs to fibronectin. ${ }^{51,52}$ Such SCF-induced adhesion was not observed in CMCs that expressed a mutant form of KIT with a phenylalanine substitution of the tyrosine residue 719 (KIT-Y719F). ${ }^{53}$ In contrast, another mutant KIT with a phenylalanine substitution of the tyrosine residue 821 (KIT-Y821F) normally responded to SCF in this adhesion. We established four IC-2 clones that expressed KIT-Y719F alone (IC-2 ${ }^{\text {KIT-Y719F), }}$

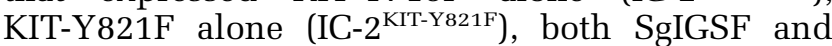

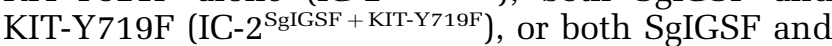

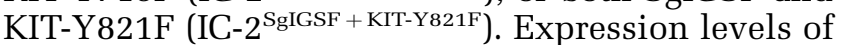
KIT of these four clones were comparable to those of either IC-2 ${ }^{\text {KIT-WT }}$ or IC-2 $2^{\text {SgIGSF + KIT-WT }}$ cells (Figure 2b). Expression levels of SgIGSF were also comparable

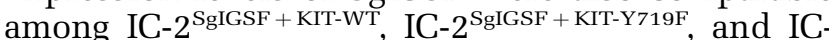
$2^{\text {SgIGSF }+ \text { KIT-Y821F }}$ cells (Figure 2b). IC-2 ${ }^{\text {KIT-Y719F }}$ and IC-2 $2^{\text {KIT-Y821F }}$ cells adhered to NIH/3T3 fibroblasts as efficiently as IC-2 $2^{\text {KIT-WT }}$ cells (Table 5). The adhesion level of IC-2 $2^{\text {SgIGSF }+ \text { KIT-Y821F }}$ cells was significantly higher than that of IC-2 ${ }^{\text {KIT-Y821F }}$ cells and was 
comparable to that of IC-2 $2^{\text {SgIGSF + KIT-WT }}$ cells (Table 5). In contrast, IC-2 $2^{\text {SgIGSF }+ \text { KIT-Y719F }}$ cells did not show the higher adhesion level when compared to that of IC-2 ${ }^{\text {KIT-Y719F }}$ cells (Table 5).

\section{Colocalization of SgIGSF and KIT in IC-2 ${ }^{\text {SgIGSF + KIT-WT }}$ Cells}

The cocultured IC-2 $2^{\text {SgIGSF }+ \text { KIT-WT }}$ and NIH/3T3 cells were stained with the anti-SgIGSF and anti-KIT Abs. Signals for either SgIGSF or KIT were detected mainly on the peripheral margin of CMCs (Figure 3). The SgIGSF signals distributed in a relatively restricted area, and colocalized well with the KIT signals (Figure 3). NIH/3T3 fibroblasts were negative for both SgIGSF and KIT (Figure 3).

\section{Discussion}

We examined the expression levels of SgIGSF and KIT in various types of mast cells and their adhesion levels to NIH/3T3 fibroblasts. Mast cells expressing
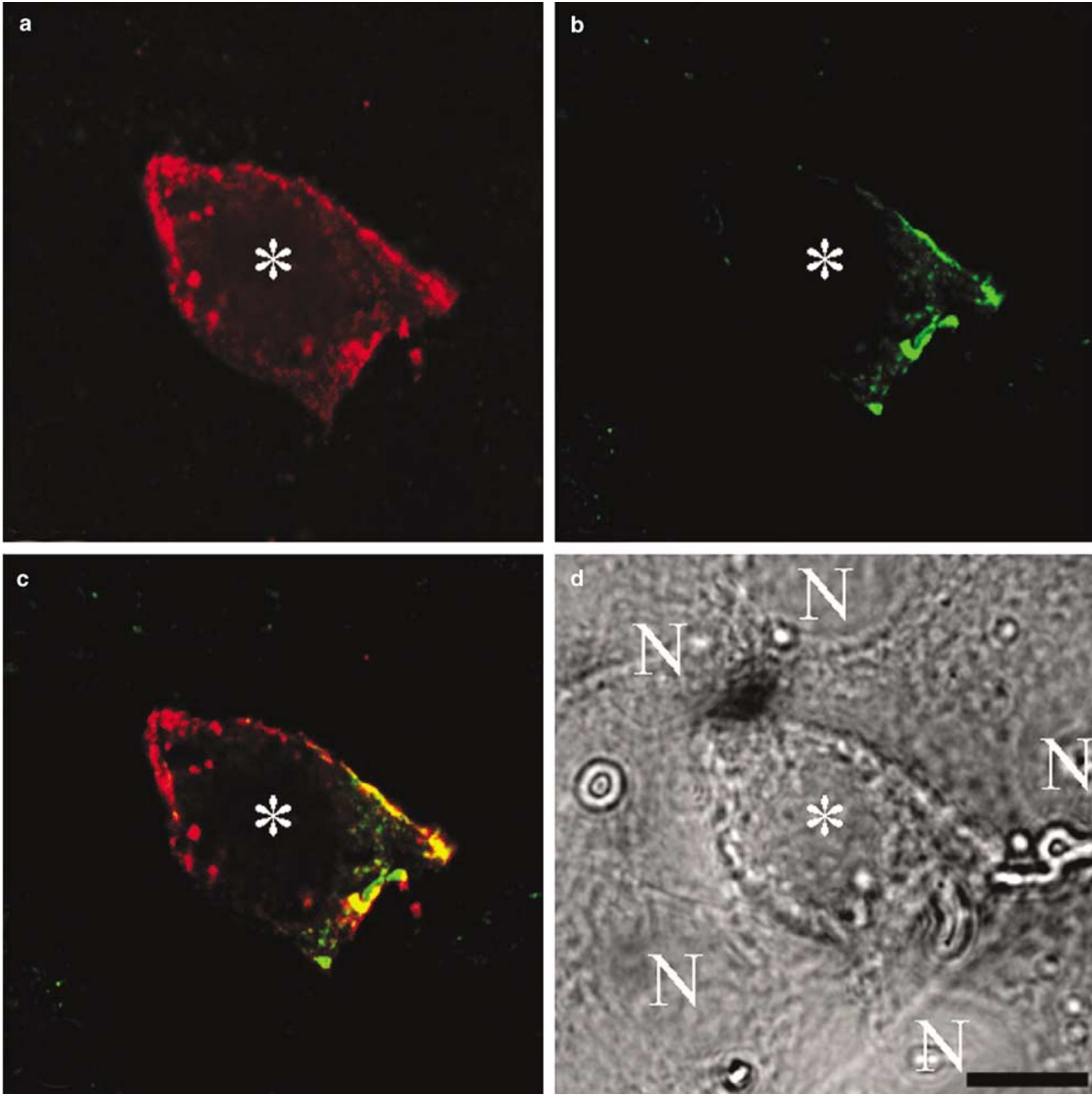

Figure 3 Colocalization of SgIGSF with KIT in the peripheral margin of WB- + / + CMC attaching to NIH/3T3 fibroblasts. The coculture of WB- + / + CMCs with NIH/3T3 fibroblasts was fixed with methanol, reacted with a mixture of the anti-KIT (H2C7) and anti-SgIGSF (rabbit polyclonal) Abs, and then stained with Cy3- (a) and Cy2- (b) conjugated secondary Abs, respectively. Cy3 and Cy2 images were merged (c). The coculture was also visualized using a differential interference contrast light microscope (d). ${ }^{*}, \mathrm{WB}-+/+\mathrm{CMC}$; N, nuclei of NIH/3T3 fibroblasts. Bar, $10 \mu \mathrm{m}$. 
both SgIGSF and KIT, such as WB- $+/+$ CMCs and P-815 cells, showed a higher adhesion level, whereas mast cells expressing neither SgIGSF nor KIT, such as IC-2 and MC/9 cells, showed a lower adhesion level. Consistent with our previous reports, ${ }^{25,54}$ both SgIGSF and KIT appeared to be involved in the adhesion of mast cells to NIH/ 3T3 fibroblasts. However, it was still unclear whether each molecule plays a distinct role in this adhesion.

We considered IC-2 cells a useful tool for investigation of the role of SgIGSF and KIT in this adhesion, because they expressed neither molecule and showed the lowest adhesion level. We expressed either SgIGSF or KIT, or both ectopically in IC-2 cells. Ectopic expression of KIT-WT significantly improved the adhesion of IC-2 cells, whereas ectopic expression of SgIGSF did not improve it at all. However, when both SgIGSF and KIT-WT were coexpressed, the adhesion level of IC-2 cells was increased more markedly than was achieved by KITWT alone. These results indicated that (1) KIT-WT by itself increased the adhesion level of IC-2 cells whereas SgIGSF by itself did not, but (2) when coexpressed with KIT-WT, SgIGSF did contribute to the adhesion of IC-2 cells; the contribution of SgIGSF was additive to that of KIT-WT. The latter notion was supported by the transfection experiment using CMCs, because ectopic SgIGSF functioned as an adhesion molecule in WB-tg/tg CMCs, which express a reduced but appreciable level of KIT.

Both SgIGSF-ED-Fc and ACK45 did inhibit the adhesion of IC-2 ${ }^{\text {SgIGSF + KIT-WT }}$ cells, but there was a large difference in the magnitude of inhibition between the two proteins. SgIGSF-ED-Fc just reduced the adhesion level of IC-2 ${ }^{\text {SgIGSF + KIT-WT }}$ cells to that of IC- $2^{\text {KIT-WT }}$ cells. Consistently, the recombinant protein lowered the adhesion level of $\mathrm{WB}-+/+$ CMCs significantly, but did not reduce it to that of WB- $W / W$ CMCs. In addition, the protein had no inhibitory effect on the adhesion of WB- $W / W$ CMCs. These results indicated again that the contribution of SgIGSF to the adhesion was additive to that of KIT-WT. In contrast, ACK45 completely abolished the contribution of both SgIGSF and KIT-WT to the adhesion. Since ACK45 not only binds to KIT but also blocks the activation of KIT by SCF, ${ }^{44,45}$ there was a possibility that intracellular signals transduced through activated KIT were necessary for SgIGSF to contribute to the adhesion of IC-2 cells.

To examine this possibility, we used two mutant forms of KIT, KIT-Y719F and KIT-Y821F. Either of the mutants was expressed singly, or coexpressed with SgIGSF in IC-2 cells. Since tyrosine 719 is the only binding site of PI3-K in KIT, KIT-Y719F is incapable of activating PI3-K whereas KIT-Y821F is normal in this respect. ${ }^{53}$ When expressed singly in IC-2 cells, either mutant form elevated the adhesion level of IC-2 cells as high as KIT-WT did. The increase in the adhesion level by single expression of KIT appeared to be independent of its intracellular signaling. This was consistent with the previous results of Adachi et al. ${ }^{54}$ They examined the adhesion to NIH/3T3 fibroblasts of CMCs derived from KIT mutants, $W / W$ and $W / W^{42}$ mice. KIT encoded by the $W$ allele does not express on the cell surface due to the lack of the transmembrane domain..$^{55}$ The mutant allele $W^{42}$ is a point mutation in the kinase domain. ${ }^{56}$ The extracellular domain of KIT encoded by the $W^{42}$ allele expresses normally on the cell surface but this mutant KIT is incapable of transducing intracellular signals. The adhesion of $W / W^{42}$ CMCs to NIH/3T3 fibroblasts was better than that of $W / W$ CMCs. ${ }^{54}$ The results of Adachi et al and the present study consistently suggest that KIT itself may behave as an adhesion molecule by using its extracellular domain in the adhesion of mast cells to NIH/3T3 fibroblasts.

When either of the two mutants was coexpressed with SgIGSF in IC-2 cells, they showed different effects on SgIGSF. Coexpression of KIT-Y821F with SgIGSF as well as that of KIT-WT with SgIGSF induced the additive effect of SgIGSF on the adhesion of IC-2 cells, whereas coexpression of KIT-Y719F with SgIGSF did not. Induction of the additive effect of SgIGSF by coexpression of KIT appeared to be dependent on intracellular signaling of KIT. This function of KIT was already suggested by several previous studies on adhesion of CMCs to extracellular matrix. ${ }^{51,52,57}$ Adhesion of CMCs to fibronectin is mediated by integrins, such as VLA5 , a complex of $\alpha 5$ and $\beta 1$ integrins expressing on CMCs. ${ }^{58}$ SCF promotes this adhesion, because in response to SCF, integrins increase their affinity to fibronectin by changing the conformation. This conformation change is dependent on PI3-K activity. Activation of KIT by SCF appears to modulate the affinity of integrins through the PI3-K-mediated intracellular signaling. However, VLA-5 did not seem to participate in the adhesion of CMCs to fibroblasts, because its expression level was not reduced in WB-tg/tg CMCs. When KIT activates PI3-K, adhesion molecules expressing on mast cells, such as SgIGSF and VLA-5, may become active selectively dependent on the types of adhesion partner molecules or cells. In fact, SgIGSF and KIT showed a similar localization on the cell membrane in CMCs that adhered to NIH/3T3 fibroblasts. Close localization of both molecules may help KIT activate SgIGSF in this adhesion.

In conclusion, the present study clearly showed that SgIGSF and KIT had distinct roles in the adhesion of mast cells to NIH/3T3 fibroblasts. Interaction between mast cells and fibroblasts induces proliferation of mast cells. This response of mast cells is believed to result from SCF-KIT interaction. As revealed here, KIT-mediated intracellular signals appear not only to induce proliferation of mast cells but also to support the adhesion of mast cells by activating adhesion molecules, such as SgIGSF. This dual action of KIT-mediated signals 
may help mast cells constantly receive growth stimuli from fibroblasts.

\section{Acknowledgements}

We thank M Kohara, K Hashimoto, and T Sawamura (Osaka University Medical School) for technical assistance. This work was supported by grants from the Ministry of Education, Culture, Sports, Science and Technology of Japan; and the Osaka Cancer Research Association.

\section{References}

1 Kitamura Y, Go S, Hatanaka K. Decrease of mast cells in $W / W^{v}$ mice and their increase by bone marrow transplantation. Blood 1978;52:447-452.

2 Kitamura Y, Go S. Decreased production of mast cells in $S 1 / S 1^{d}$ anemic mice. Blood 1979;53:492-497.

3 Chabot B, Stephenson DA, Chapman VM, et al. The proto-oncogene c-kit encoding a transmembrane tyrosine kinase receptor maps to the mouse $W$ locus. Nature 1988;335:88-89.

4 Geissler EN, Ryan MA, Housman DE. The dominantwhite spotting $(W)$ locus of the mouse encodes the c-kit proto-oncogene. Cell 1988;55:185-192.

5 Nocka K, Majumder S, Chabot B, et al. Expression of c-kit gene products in known cellular targets of $W$ mutations in normal and $W$ mutant mice-evidence for an impaired c-kit kinase in mutant mice. Genes Dev 1989;3:816-826.

6 Williams DE, Eisenman J, Baird A, et al. Identification of a ligand for the c-kit proto-oncogene. Cell 1990; 63:167-174.

7 Zsebo KM, Williams DA, Geissler EN, et al. Stem cell factor is encoded at the SI locus of the mouse and is the ligand for the c-kit tyrosine kinase receptor. Cell 1990;63:213-224.

8 Huang E, Nocka K, Beier DR, et al. The hematopoietic growth factor KL is encoded by the $S l$ locus and is the ligand of the c-kit receptor, the gene product of the $W$ locus. Cell 1990;63:225-233.

9 Flanagan JG, Chan DC, Leder P. Transmembrane form of the kit ligand growth factor is determined by alternative splicing and is missing in the $S l^{d}$ mutant. Cell 1991;64:1025-1035.

10 Flanagan JG, Leder P. The kit ligand: a cell surface molecule altered in steel mutant fibroblasts. Cell 1990;63:185-194.

11 Galli SJ, Zsebo KM, Geissler EN. The kit ligand, stem cell factor. Adv Immunol 1994;55:1-96.

12 Levi-Schaffer $\mathrm{F}$, Austen KF, Gravallese PM, et al. Coculture of interleukin 3-dependent mouse mast cells with fibroblasts results in a phenotypic change of the mast cells. Proc Natl Acad Sci USA 1986;83:64856488.

13 Fujita J, Nakayama $\mathrm{H}$, Onoue $\mathrm{H}$, et al. Fibroblastdependent growth of mouse mast cells in vitro: duplication of mast cell depletion in mutant mice of $W / W^{v}$ genotype. J Cell Physiol 1988;134:78-84.

14 Fujita J, Nakayama $\mathrm{H}$, Onoue $\mathrm{H}$, et al. Failure of $W / W^{v}$ mouse-derived cultured mast cells to enter $S$ phase upon contact with NIH/3T3 fibroblasts. Blood 1988; 72:463-468.

15 Nocka K, Tan JC, Chiu E, et al. Molecular bases of dominant negative and loss of function mutations at the murine c-kit/white spotting locus: $W^{37}, W^{v}, W^{41}$ and $W$. EMBO J 1990;9:1805-1813.

16 Reith AD, Rottapel R, Giddens E, et al. $W$ mutant mice with mild or severe developmental defects contain distinct point mutations in the kinase domain of the c-kit receptor. Genes Dev 1990;4:390-400.

17 Morii E, Ogihara H, Oboki K, et al. Effect of a large deletion of the basic domain of $m i$ transcription factor on differentiation of mast cells. Blood 2001;98:25772579.

18 Kim DK, Morii E, Ogihara H, et al. Different effect of various mutant MITF encoded by $m i, M i^{\text {or }}$, or $M i^{\text {wh }}$ allele on phenotype of murine mast cells. Blood 1999;93:4179-4186.

19 Morii E, Ogihara H, Kim DK, et al. Importance of leucine zipper domain of $\mathrm{mi}$ transcription factor (MITF) for differentiation of mast cells demonstrated using $m i^{\mathrm{ce}} / \mathrm{mi}^{c e}$ mutant mice of which MITF lacks the zipper domain. Blood 2001;97:2038-2044.

20 Kataoka TR, Morii E, Oboki K, et al. Dual abnormal effects of mutant MITF encoded by $\mathrm{Mi}^{\text {wh }}$ allele on mouse mast cells: decreased but recognizable transactivation and inhibition of transactivation. Biochem Biophys Res Commun 2002;297:111-115.

21 Hodgkinson CA, Moore KJ, Nakayama A, et al. Mutations at the mouse microphthalmia locus are associated with defects in a gene encoding a novel basic-helix-loop-helix-zipper protein. Cell 1993;74: 395-404.

22 Hughes JJ, Lingrel JB, Krakowsky JM, et al. A helix-loop-helix transcription factor-like gene is located at the mi locus. J Biol Chem 1993;268: 20687-20690.

23 Tachibana M, Hara Y, Vyas D, et al. Cochlear disorder associated with melanocyte anomaly in mice with a transgenic insertional mutation. Mol Cell Neurosci 1992;3:433-445.

24 Tsujimura T, Hashimoto K, Morii E, et al. Involvement of transcription factor encoded by the mouse mi locus (MITF) in apoptosis of cultured mast cells induced by removal of interleukin-3. Am J Pathol 1997;151: 1043-1051.

25 Ito A, Jippo T, Wakayama T, et al. SgIGSF: a new mastcell adhesion molecule used for attachment to fibroblasts and transcriptionally regulated by MITF. Blood 2003;101:2601-2608.

26 Besmer P, Murphy JE, George PC, et al. A new acute transforming feline retrovirus and relationship of its oncogene v-kit with the protein kinase gene family. Nature 1986;320:415-421.

27 Yarden Y, Kuang WJ, Yang-Feng T, et al. Human protooncogene c-kit: a new cell surface receptor tyrosine kinase for an unidentified ligand. EMBO J 1987;6: 3341-3351.

28 Qiu FH, Ray P, Brown K, et al. Primary structure of ckit: relationship with the CSF-1/PDGF receptor kinase family-oncogenic activation of v-kit involves deletion of extracellular domain and $\mathrm{C}$ terminus. EMBO J 1988;7:1003-1011.

29 Wakayama T, Ohashi K, Mizuno K, et al. Cloning and characterization of a novel mouse immunoglobulin superfamily gene expressed in early spermatogenic cells. Mol Reprod Dev 2001;60:158-164. 
30 Yageta M, Kuramochi M, Masuda M, et al. Direct association of TSLC1 and DAL-1, two distinct tumor suppressor proteins in lung cancer. Cancer Res 2002; 62:5129-5133.

31 Koyasu S, Nakauchi H, Kitamura K, et al. Production of interleukin 3 and gamma-interferon by an antigenspecific mouse suppressor $\mathrm{T}$ cell clone. J Immunol 1985;134:3130-3136.

32 Nabel G, Galli SJ, Dvorak AM, et al. Inducer T lymphocytes synthesize a factor that stimulates proliferation of cloned mast cells. Nature 1981;291: 332-334.

33 Furth J, Hagen P, Hirsch EI. Transplantable mastocytoma in the mouse containing histamine, heparin, 5-hydroxytryptamine. Proc Soc Exp Biol Med 1957; 95:824-828.

34 Dunn TB, Potter M. A transplantable mast-cell neoplasm in the mouse. J Natl Cancer Inst 1957;18: 587-601.

35 Montgomery RI, Lidholt K, Flay NW, et al. Stable heparin-producing cell lines derived from the Furth murine mastocytoma. Proc Natl Acad Sci USA 1992; 89:11327-11331.

36 Mann R, Mulligan RC, Baltimore D. Construction of a retrovirus packaging mutant and its use to produce helper-free defective retrovirus. Cell 1983;33:153-159.

37 Morita S, Kojima T, Kitamura T. Plat-E: an efficient and stable system for transient packaging of retroviruses. Gene Therapy 2000;7:1063-1066.

38 Wakayama T, Koami H, Ariga H, et al. Expression and functional characterization of the adhesion molecule spermatogenic immunoglobulin superfamily in the mouse testis. Biol Reprod 2003;68:1755-1763.

39 Yamatani H, Sato Y, Fujisawa H, et al. Chronotopic organization of olfactory bulb axons in the lateral olfactory tract. J Comp Neurol 2004;475: 247-260.

40 Koma Y, Ito A, Wakayama T, et al. Cloning of a soluble isoform of the SgIGSF adhesion molecule that binds the extracellular domain of the membrane-bound isoform. Oncogene 2004;23:5687-5692.

41 Ueda S, Mizuki M, Ikeda H, et al. Critical roles of c-Kit tyrosine residues 567 and 719 in stem cell factorinduced chemotaxis: contribution of src family kinase and PI3-kinase on calcium mobilization and cell migration. Blood 2002;99:3342-3349.

42 Laker C, Stocking C, Bergholz U, et al. Autocrine stimulation after transfer of the granulocyte/macrophage colony-stimulating factor gene and autonomous growth are distinct but interdependent steps in the oncogenic pathway. Proc Natl Acad Sci USA 1987; 84:8458-8462.

43 Koshimizu U, Tsujimura T, Isozaki K, et al. $W^{n}$ mutation of c-kit receptor affects its post-translational processing and extracellular expression. Oncogene 1994;9:157-162.
44 Ogawa M, Matsuzaki Y, Nishikawa S, et al. Expression and function of c-kit in hemopoietic progenitor cells. J Exp Med 1991;174:63-71.

45 Nishikawa S, Kusakabe M, Yoshinaga K, et al. In utero manipulation of coat color formation by a monoclonal anti-c-kit antibody: two distinct waves of c-kit-dependency during melanocyte development. EMBO J 1991; 10:2111-2118.

46 Reith AD, Ellis C, Lyman SD, et al. Signal transduction by normal isoforms and $W$ mutant variants of the Kit receptor tyrosine kinase. EMBO J 1991;10:2451-2459.

47 Rottapel R, Reedijk M, Williams DE, et al. The Steel/W transduction pathway: kit autophosphorylation and its association with a unique subset of cytoplasmic signaling proteins is induced by the Steel factor. Mol Cell Biol 1991;11:3043-3051.

48 Serve H, Hsu YC, Besmer P. Tyrosine residue 719 of the c-kit receptor is essential for binding of the $\mathrm{P} 85$ subunit of phosphatidylinositol (PI) 3-kinase and for c-kit-associated PI 3-kinase activity in COS-1 cells. J Biol Chem 1994;269:6026-6030.

49 Roussel MF, Shurtleff SA, Downing JR, et al. A point mutation at tyrosine-809 in the human colony-stimulating factor 1 receptor impairs mitogenesis without abrogating tyrosine kinase activity, association with phosphatidylinositol 3-kinase, or induction of c-fos and junB genes. Proc Natl Acad Sci USA 1990;87: 6738-6742.

50 Roussel MF, Cleveland JL, Shurtleff SA, et al. Myc rescue of a mutant CSF-1 receptor impaired in mitogenic signalling. Nature 1991;353:361-363.

51 Dastych J, Metcalfe DD. Stem cell factor induces mast cell adhesion to fibronectin. J Immunol 1994;152: 213-219.

52 Kinashi T, Springer TA. Steel factor and c-kit regulate cell-matrix adhesion. Blood 1994;83:1033-1038.

53 Serve H, Yee NS, Stella G, et al. Differential roles of PI3-kinase and Kit tyrosine 821 in Kit receptormediated proliferation, survival and cell adhesion in mast cells. EMBO J 1995;14:473-483.

54 Adachi S, Ebi Y, Nishikawa S, et al. Necessity of extracellular domain of $W$ (c-kit) receptors for attachment of murine cultured mast cells to fibroblasts. Blood 1992;79:650-656.

55 Hayashi S, Kunisada T, Ogawa M, et al. Exon skipping by mutation of an authentic splice site of c-kit gene in $W / W$ mouse. Nucleic Acids Res 1991;19:1267-1271.

56 Tan JC, Nocka K, Ray $\mathrm{P}$, et al. The dominant $W^{42}$ spotting phenotype results from a missense mutation in the c-kit receptor kinase. Science 1990;247:209-212.

57 Kinashi T, Springer TA. Adhesion molecules in hematopoietic cells. Blood Cells 1994;20:25-44.

58 Kinashi T, Asaoka T, Setoguchi R, et al. Affinity modulation of very late antigen-5 through phosphatidylinositol 3-kinase in mast cells. J Immunol 1999; 162:2850-2857. 